\title{
Current Status of Computed Tomography in Novel Coronavirus Disease 2019 Pneumonia
}

\author{
Kumble Seetharama Madhusudhan ${ }^{1}$ Deep Narayan Srivastava ${ }^{1}$ \\ ${ }^{1}$ Department of Radiodiagnosis, All India Institute of Medical \\ Sciences, New Delhi, India

\begin{abstract}
Address for correspondence Kumble Seetharama Madhusudhan, MD, FRCR, Department of Radiodiagnosis, All India Institute of Medical Sciences, Ansari Nagar, New Delhi 110029, India (e-mail:drmadhuks@gmail.com).
\end{abstract}

Ann Natl Acad Med Sci (India):2020;2:62-66

\begin{abstract}
Keywords

- severe acute respiratory syndrome

- coronavirus disease

- COVID-19

- computed tomography

- consolidation

- ground glass opacity

The novel coronavirus disease, known as severe acute respiratory syndrome coronavirus 2 (SARS-CoV2), has developed into a pandemic affecting more than three million people worldwide. It predominantly affects the respiratory system and patients present with fever, dry cough, dyspnea, and myalgia. The confirmatory diagnostic test is real-time reverse transcriptase polymerase chain reaction on blood or respiratory samples. Imaging with computed tomography, although not routinely recommended, may not only assist in making a diagnosis but also in assessing disease progression, assessing complications, and in prognostication. This review describes the objectives, techniques, imaging features, and reporting of computed tomography findings of SARS-CoV2 pneumonia.
\end{abstract}

\section{Introduction}

The novel coronavirus, which causes severe acute respiratory syndrome (SARS) like illness, is a newly found zoonotic virus originating from the bats. ${ }^{1}$ This virus, now called, SARS coronavirus-2 (SARS-CoV) belongs to the subgenus Sarbecovirus and family Coronaviridae. ${ }^{2}$ These are single-stranded RNA viruses and bats are natural hosts and reservoirs. ${ }^{2}$ After the initial outbreak in China in late December 2019, the disease has spread worldwide with over 3 million cases infected and over 200,000 dead in just 4 months and numbers continue to rise. ${ }^{3}$

The SARS-CoV2 virus predominantly affects the respiratory system and the symptoms differ from the other diseases caused by coronavirus family like SARS and Middle East Respiratory Syndrome (MERS). ${ }^{4}$ The disease affects all ages. About 37\% of the infected patients had associated comorbidities such as hypertension, cardiovascular disease, and diabetes mellitus. ${ }^{5}$ The common clinical manifestations include fever, dry cough, dyspnea, and myalgia. ${ }^{4,5}$ Laboratory findings are nonspecific with elevation of C-reactive protein, erythrocyte sedimentation rate, and lactate dehydrogenase. ${ }^{5}$

In view of the highly contagious nature of the disease and the absence of any definitive treatment, early diagnosis is necessary to isolate the infected person. ${ }^{6}$ The diagnosis is confirmed by reverse-transcriptase polymerase chain reaction (RT-PCR) on the respiratory or blood samples by identifying the RNA gene. However, it has a sensitivity over $90 \%{ }^{7,8}$ The real-time RT-PCR takes 5 to 6 hours to provide the final result. ${ }^{9}$ Radiological imaging is a critical component of the diagnostic workup, monitoring of disease progression, and follow-up in coronavirus-related pulmonary syndromes. Although initially it was suggested that imaging, particularly with computed tomography (CT) scan, plays an important role in the diagnosis of SARS-CoV2 infection, its specificity is low (25\%) and may be normal ( - Fig. 1) ${ }^{6,7}$ The typical imaging features of coronavirus disease 2019 (COVID-19) pneumonia are also nonspecific and can be seen in many other diseases. ${ }^{10}$ Hence, it is not routinely recommended.

\section{Pathology}

Although most patients have mild symptoms, lung involvement is found in most patients, even in those without symptoms. ${ }^{11}$ The pathological changes in the lungs in SARS-CoV2 are found to be similar to that of SARS and MERS. ${ }^{12,13}$ The main pulmonary histological findings include deposition of hyaline membrane, damage to the alveolar 
epithelial cells, exudates of fibrin, and diffuse hyperplasia of type II pneumocytes, all of which are the features of diffuse alveolar damage. ${ }^{14}$ The disease progresses from mild exudative phase involving alveolar edema, proteinaceous exudate, focal pneumocyte hyperplasia, and patchy infiltration of inflammatory cells to consolidation, when there is proliferation of fibroblasts with clusters of extracellular matrix and fibrin within the alveoli (-Figs. 2 and 3). ${ }^{14}$ These changes match with the imaging findings of ground glass opacities (GGOs) and consolidation. Intra-alveolar neutrophilic infiltration may be seen in the event of a superimposed bacterial bronchopneumonia. The preferential involvement of lungs in most cases has been attributed to the presence of high concentration of angiotensin converting enzyme 2 within the pneumocytes that are specifically utilized by the virus. ${ }^{15}$

\section{Precautions While Performing CT Scan}

All CT scans when done for a SARS-CoV2 positive patient should be done by a technologist after donning the personal protective equipment. ${ }^{16}$ Two technologists are needed for performing a CT scan, one to position the patient and the other in the console room. ${ }^{17}$ Entry of personnel into the CT room should be restricted. The scans should be properly scheduled to later part of the day after the routine other cases, unless it is an emergency.

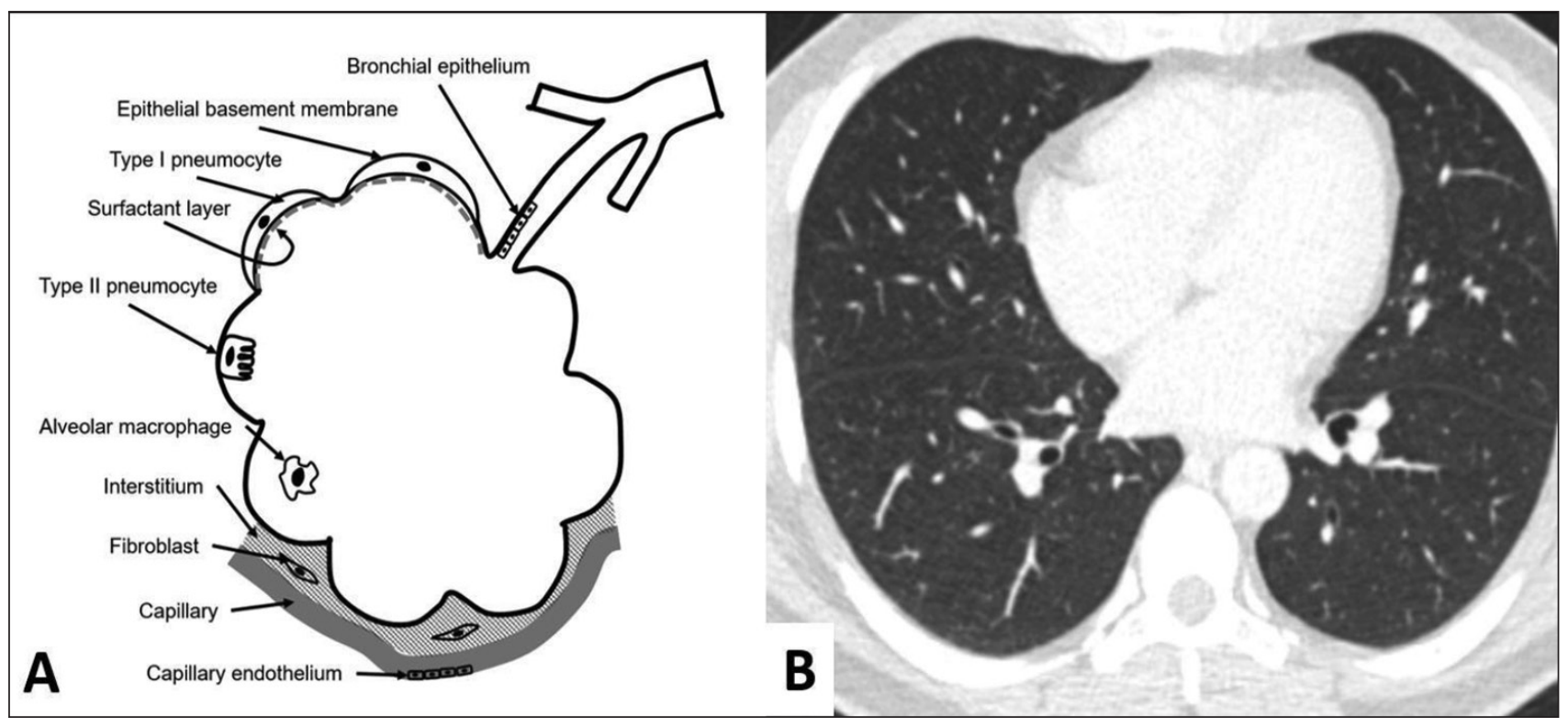

Fig. 1 Normal appearance. (A) Schematic diagram of a terminal alveolus showing normal structures. (B) Computed tomography scan (lung window) of normal lungs.

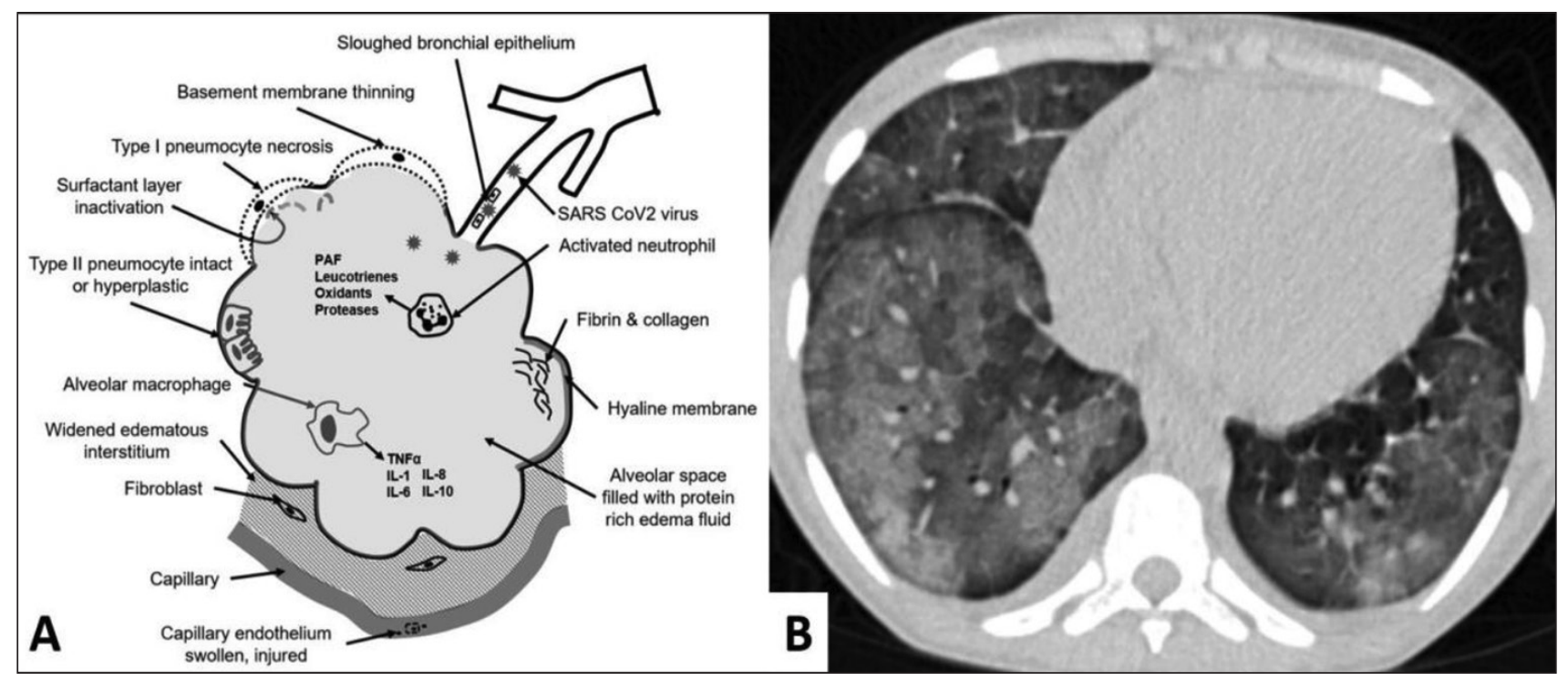

Fig. 2 Early exudative phase. (A) Schematic diagram showing changes at the level of alveolus in the early phase. (B) Axial computed tomography scan (lung window) showing ground glass opacities in both lungs. 


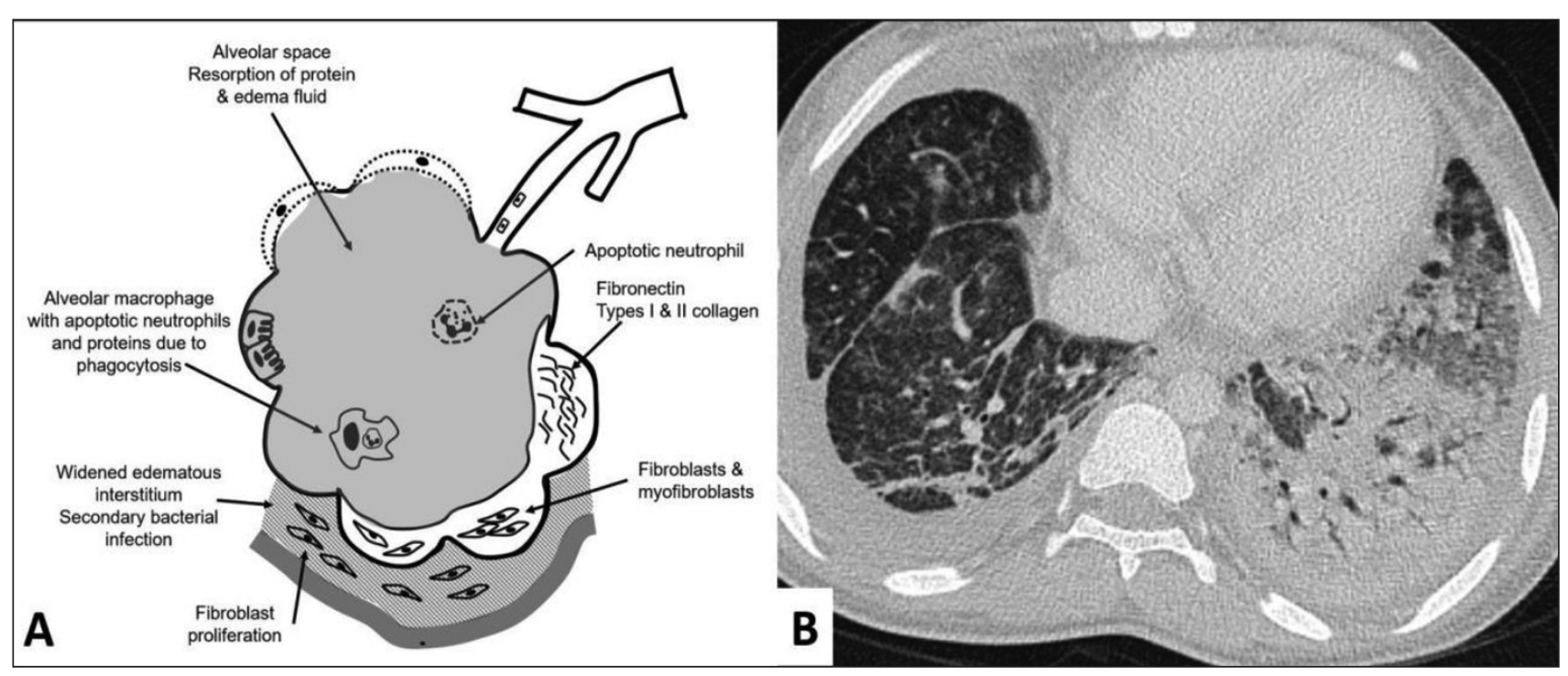

Fig. 3 Late exudative phase. (A) Schematic diagram showing changes at the level of alveolus in the late phase. (B) Axial computed tomography scan (lung window) showing consolidation with air bronchogram in the left lower lobe.

After the CT scans have been performed, the CT room should be properly sanitized. ${ }^{17}$ The high-frequency contact surfaces should be wiped with a cloth soaked in alcohol-based disinfectants. The protocol for decontamination is to mop the floor and to wipe the CT scanner, initially with water mixed with detergent followed by sodium hypochlorite solution. Subsequently, the room is fumigated with formaldehyde for 30 minutes. The CT suite should be completely closed for 1 hour and properly ventilated.

\section{Imaging Features on Computed Tomography}

The noncontrast $\mathrm{CT}$ scan of the chest is performed using the standard technique. The patient is in supine position and the scan is performed from the level of thoracic inlet to the level of costophrenic angles, with breath held in end-expiration. ${ }^{11}$ Multidetector CT scan is preferred with images reconstructed at slice thickness of $1.5 \mathrm{~mm}$. Intravenous contrast is usually not required. ${ }^{18}$ The iodinated contrast enhanced CT is done in specific situations like hemoptysis or when we need to see pulmonary vasculature. Both lung and mediastinal windows should be evaluated along with multiplanar reconstructions.

Various findings on CT have been described that assist in the diagnosis of SARS-CoV2 pneumonia. These can be broadly classified into alveolar or parenchymal, airway, pleural, and other organ changes. $6,11,19,20$

\section{Alveolar/Parenchymal Changes}

GGO with ill-defined margins is the most common manifestation of SARS-CoV2 pneumonia (- Fig. 2). ${ }^{11,21}$ It is considered as one of the earliest signs and is present in up to 98\% of the patients. ${ }^{22,23}$ Consolidation is another common finding ( - Fig. 3). ${ }^{14,19}$ The consolidation may be found de novo or may develop in an area of previous GGO, indicating that this is seen more commonly as the disease progresses. ${ }^{11,22,24}$ It may be associated with an air-bronchogram ( - Fig. 3 ). Consolidations are more commonly seen in late stage of the disease and in patients older than 50 years and hence, may be an indication of more severe disease..$^{24}$ Further, many of the patients have a combination of GGO and consolidation during the course of the disease. A reverse halo or atoll sign has also been described when a ring of consolidation develops around an area of GGO. ${ }^{10}$ Round cystic changes might be associated with the process of resorption of consolidation, which might be explained by the infection causing damage to the alveolar walls and leading to pneumatoceles. ${ }^{11}$ Enlargement of the vessels is another common finding seen in $~ 70 \%$ of the patients. ${ }^{20}$ This finding is typically seen within or around an area of GGO or consolidation and thought to be due to the acute inflammation resulting in injury and edema of the vessel wall. ${ }^{19,20}$

Along with alveolar infiltration, there may be an associated infiltration of the interstitial tissue in $49 \%{ }^{11,19,20} \mathrm{~A}$ finding described with this feature is in 5 to $36 \%$ crazy paving pattern, which occurs when there is a combination of GGO and interlobular septal thickening. ${ }^{10}$ Along with consolidation, this finding may be an indication of progressive disease. ${ }^{25}$ Centrilobular nodules have been found in 23\% of the patients. ${ }^{20}$ They may be solitary or multiple and have ill-defined margins. ${ }^{19}$ These nodules may increase in size and number as the disease progresses. ${ }^{26}$

Fibrosis and architectural distortion is a finding (21-53\%) seen in SARS-CoV2 pneumonia and indicates the healing process of the GGO, consolidation, and interstitial disease. ${ }^{18,20,26}$ Fibrosis may be in the airspace or interstitium or both and results in distortion of the parenchymal architecture, volume loss, and mediastinal shifts. ${ }^{18}$ The prognosis of the fibrosis is still not clear. It is too early to suggest if the fibrosis is irreversible.

The distribution of the findings in the pulmonary parenchyma has been described in most of the studies. Lower lobes of the lungs are the most common sites of involvement, found in $55 \%$ of the cases ( - Fig. 3). ${ }^{20}$ Upper lobes are involved in $6 \%$ and in the remaining, no craniocaudal distribution is 
seen. Within a lobe, most common site is peripheral subpleural regions, seen in more than $75 \%$ of the cases. ${ }^{20,22}$ Bilateral lungs are affected in more than $80 \%$ and it was commonly multifocal ( $\sim 55 \%)$ ( - Fig. 2). In early phase, it may present as a unifocal lesion, commonly located in the inferior lobe of the right lung. ${ }^{11,27}$

\section{Airway Changes}

Bronchial wall thickening is an uncommon finding, seen in less than a third of patients. ${ }^{20}$ It has been found that bronchial wall thickening was more common in severe and critically ill patients and may have a prognostic significance. ${ }^{23}$ Bronchiectasis or bronchiolectasis occurs either due to bronchial wall inflammation or due to surrounding fibrosis developing during the healing phase (tractional bronchiectasis). ${ }^{19}$ It is found as the diseases progresses into the second and third week and may indicate the onset of healing process. ${ }^{11}$ A small air-containing space has been variably labeled as cystic change, cavity sign, and air-bubble sign. ${ }^{19}$ This could be due to bronchiolectasis or resorption of an area of consolidation.

\section{Pleural Changes}

Pleural effusion is an uncommon finding seen in 5 to $14 \%$ of the cases. ${ }^{11,20}$ Pleural effusion develops in the late stage of the disease and may represent poor prognosis. ${ }^{18}$ Thickening of the pleura is a more common finding, found in one third of patients. ${ }^{11}$ This is usually seen in association with underlying lung involvement in the form of GGO, consolidation, or interstitial thickening. Subpleural lines or bands are seen in less than a third of patients. They are seen as curvilinear density of 1 to $3 \mathrm{~mm}$ in thickness, located $<1 \mathrm{~cm}$ from the pleural surface. ${ }^{19}$ This finding may be related to either pulmonary edema or fibrosis. ${ }^{23}$

\section{Other Changes}

Lymphadenopathy is an uncommon finding in SARS-CoV2 pneumonia. It is reported in 4 to $8 \%$ of patients. ${ }^{11}$ It is commonly found in patients with severe and critical disease and thus may be a poor prognostic factor. ${ }^{23}$ Pericardial effusion is found in $\sim 5 \%$ of the patients and is more commonly seen with progression of the disease. ${ }^{22}$ Similar to lymphadenopathy, it may also carry poorer prognosis. ${ }^{23}$

Some studies have shown perfusion abnormalities on dual energy CT and suggested that processes other than alveolar damage might be involved in hypoxemia related to COVID19 pneumonia. Further details of the vascular and perfusion changes in patients with COVID-19 are yet to be assessed. ${ }^{28}$

\section{Disease Evolution}

Healing usually begins after 14 days of illness in $~ 75 \%$ of cases. ${ }^{22}$ The imaging findings gradually improve with decrease in the extent of lobar involvement, resorption of consolidation, and disappearance of crazy-paving feature..$^{25}$ Later on, there may be cavitation, development of fibrosis and bronchiectasis. It is currently not clear how the fibrosis progresses.

\section{Differential Diagnosis}

Since SARS-CoV2 belongs to Coronaviridae family, other diseases caused by this family like SARS and MERS are the common differentials. Findings on CT scan are similar in all these three diseases. ${ }^{29}$ However, some of the findings are found to be more common in SARS-CoV2 compared with MERS and SARS. These include predominant multifocal involvement, nodules with halo sign, and reverse halo sign. ${ }^{29}$ The latter two findings have not been described in MERS and SARS and may be relatively specific to SARS-CoV2.

\section{Current Status of CT Scan for SARS-CoV2 Infection}

The RT-PCR test for SARS-CoV2 infection is highly sensitive with sensitivity rates ranging from 93 to $100 \%{ }^{30}$ The results are usually available within 6 to 12 hours, and within a day in all of the patients. The CT scan has a sensitivity and specificity in the range of 80 to $90 \%$ and 60 to $70 \%$, respectively, for the diagnosis of SARS-CoV2 infection. ${ }^{6}$ Out of the patients who were RT-PCR positive, the CT scan showed findings in 61 to $97 \%$ in various studies. ${ }^{31}$

Since abnormal lung CT findings can be present even in asymptomatic patients, CT scan may have a role in a clinically suspected case with negative RT-PCR. ${ }^{23} \mathrm{CT}$ scan currently has not been recommended for routine use in the diagnosis of SARS-CoV2 pneumonia. Further, routine use of CT scan will overwhelm the resources and will reduce the throughput for non-COVID cases as the machine will be closed frequently for sanitization. The radiology team should be aware of all precautions and strategies to minimize the risk of infection among staff and patients. ${ }^{32}$

Since the chest imaging is an important component of patient management in individuals with COVID-19, further investigations are required to expand understanding of the imaging findings throughout the disease course. ${ }^{32}$ The experiences with SARS and MERS show that follow-up imaging should be performed in individuals recovering from COVID-19 to look for evidence of chronic involvement of the lungs (i.e., interlobular thickening, air trapping or fibrosis). ${ }^{33}$

\section{Conclusion}

SARS-CoV2 is a zoonotic viral disease predominantly involving the respiratory system. The real-time RT-PCR is highly sensitive and is diagnostic in $>95 \%$ cases. Routine imaging of the chest with CT scan for diagnosis is not currently indicated. In the current prevalence of the disease, incidental detection of COVID-19 pneumonia on CT examinations not directly performed for this has critical importance since radiologically visible COVID-19 pneumonia is associated with possible viral transmission. ${ }^{34}$ However, early reports have stated that initial imaging might show normal findings in $15 \%$ of individuals, so a normal chest imaging examination does not exclude the infection. ${ }^{33}$ Knowledge of the spectrum of findings on the CT scan, although non-specific, may assist the radiologist to suggest a diagnosis if found incidentally in non-suspected patients. Using CT diagnostically is not known to provide clinical benefit(s) and could lead to false security 
if results are negative. ${ }^{35}$ The objective quantification of lung involvement manually or by artificial intelligence at the time of diagnosis might have prognostic value and an effect on the choice of therapy. ${ }^{36}$

\section{Conflict of Interest}

None declared.

\section{References}

1 Bonilla-Aldana DK, Dhama K, Rodriguez-Morales AJ. Revisiting the one health approach in the context of COVID-19: a look into the ecology of this emerging disease. Adv Anim Vet Sci 2020;8:234-237

2 Zhu N, Zhang D, Wang W, et al; China Novel Coronavirus Investigating and Research Team. A Novel coronavirus from patients with pneumonia in China, 2019. N Engl J Med 2020;382(8):727-733

3 WHO coronavirus disease (COVID-19) situation dashboard. Available at: https://covid19.who.int/. Accessed May 22, 2020

4 Huang C, Wang Y, Li X, et al. Clinical features of patients infected with 2019 novel coronavirus in Wuhan, China. Lancet 2020;395(10223):497-506

5 Rodriguez-Morales AJ, Cardona-Ospina JA, Gutiérrez-Ocampo E, et al; Latin American Network of Coronavirus Disease 2019COVID-19 Research (LANCOVID-19). Electronic address:, https://www.lancovid.org. Clinical, laboratory and imaging features of COVID-19: a systematic review and meta-analysis. Travel Med Infect Dis 2020;34:101623

6 Ai T, Yang Z, Hou H, et al. Correlation of chest CT and RT-PCR testing in coronavirus disease 2019 (COVID-19) in China: a report of 1014 cases. Radiology 2020;200642:200642

7 Fang Y, Zhang H, Xie J, et al. Sensitivity of chest CT for COVID19: comparison to RT-PCR. Radiology 2020;200432:200432

8 Long C, Xu H, Shen Q et al. Diagnosis of the coronavirus disease (COVID-19): rRT-PCR or CT? Eur J Radiol 2020;126:108961

9 Udugama B, Kadhiresan P, Kozlowski HN, et al. Diagnosing COVID-19: the disease and tools for detection. ACS Nano 2020;14(4):3822-3835

10 Bernheim A, Mei X, Huang M, et al. Chest CT findings in coronavirus disease-19 (COVID-19): relationship to duration of infection. Radiology 2020;295(3):200463

11 Shi H, Han X, Jiang N, et al. Radiological findings from 81 patients with COVID-19 pneumonia in Wuhan, China: a descriptive study. Lancet Infect Dis 2020;20(4):425-434

12 Ding Y, Wang $\mathrm{H}$, Shen $\mathrm{H}$, et al. The clinical pathology of severe acute respiratory syndrome (SARS): a report from China. J Pathol 2003;200(3):282-289

$13 \mathrm{Ng}$ DL, Al Hosani F, Keating MK, et al. Clinicopathologic, immunohistochemical, and ultrastructural findings of a fatal case of Middle East respiratory syndrome coronavirus infection in the United Arab Emirates, April 2014. Am J Pathol 2016;186(3):652-658

14 Tian S, Xiong Y, Liu H, et al. Pathological study of the 2019 novel coronavirus disease (COVID-19) through postmortem core biopsies. Mod Pathol 2020. Doi:10.1038/s41379-020-0536-X

$15 \mathrm{Xu} \mathrm{X}$, Chen P, Wang J, et al. Evolution of the novel coronavirus from the ongoing Wuhan outbreak and modeling of its spike protein for risk of human transmission. Sci China Life Sci 2020;63(3):457-460

16 Mossa-Basha M, Medverd J, Linnau K, et al. Policies and guidelines for COVID-19 preparedness: experiences from the University of Washington. Radiology 2020;201326

17 Nakajima K, Kato H, Yamashiro T, et al. COVID-19 pneumonia: infection control protocol inside computed tomography suites. Jpn J Radiol 2020;38(5):391-393
18 Li K, Fang Y, Li W, et al. CT image visual quantitative evaluation and clinical classification of coronavirus disease (COVID-19) Eur Radiol 2020. Doi: 10.1007/s00330-020-06817-6

19 Ye Z, Zhang Y, Wang Y, Huang Z, Song B. Chest CT manifestations of new coronavirus disease 2019 (COVID-19): a pictorial review. Eur Radiol 2020. Doi:10.1007/s00330-020-06801-0

20 Zhao W, Zhong Z, Xie X, Yu Q, Liu J. Relation between chest CT findings and clinical conditions of coronavirus disease (COVID-19) pneumonia: a multicenter study. Am J Roentgenol 2020;214(5):1072-1077

21 Yang Q, Liu Q, Xu H, Lu H, Liu S, Li H. Imaging of coronavirus disease 2019: a Chinese expert consensus statement. Eur J Radiol 2020;127:109008

22 Salehi S, Abedi A, Balakrishnan S, Gholamrezanezhad A. Coronavirus disease 2019 (COVID-19): a systematic review of imaging findings in 919 patients. Am J Roentgenol 2020. Doi:10.2214/AJR.20.23034

23 Kunhua Li JW, Wu F, Guo D, Chen L, Zheng F, Li C. The clinical and chest CT features associated with severe and critical COVID-19 pneumonia. Invest Radiol 2020. Doi:55(6)327-331

24 Song F, Shi N, Shan F, et al. Emerging coronavirus 2019- nCoV pneumonia. Radiology 2020;295(1):210-217

25 Pan F, Ye T, Sun P, et al. Time course of lung changes on chest CT during recovery from 2019 novel coronavirus (COVID19) pneumonia. Radiology 2020. Doi:10.1148/radiol.2020200370

26 Pan Y, Guan H, Zhou S, et al. Initial CT findings and temporal changes in patients with the novel coronavirus pneumonia (2019-nCoV): a study of 63 patients in Wuhan, China. Eur Radiol 2020. Doi:10.1007/s00330-020-06731-x

27 Zhou S, Wang Y, Zhu T, Xia L. CT features of coronavirus disease 2019 (COVID-19) pneumonia in 62 patients in Wuhan, China. Am J Roentgenol 2020;214:1-8

28 Lang M, Som A, Mendoza DP, et al. Hypoxaemia related to COVID-19: vascular and perfusion abnormalities on dual-energy CT. Lancet Infect Dis 2020. Doi: 10.1016/ S1473-3099(20)30367-4

29 Li Y, Xia L. Coronavirus disease 2019 (COVID-19): role of chest CT in diagnosis and management. Am J Roentgenol 2020. Doi;10.2214/AJR.20.22954

30 Mossa-Basha M, Meltzer CC, Kim DC, Tuite MJ, Kolli KP, Tan BS. Radiology department preparedness for COVID19:radiologyscientific expert panel. Radiology 2020. Doi;10.1148/radiol.2020200988

31 Hope MD, Raptis CA, Shah A, Hammer MM, Henry TS; six signatories. A role for CT in COVID-19? What data really tell us so far. Lancet 2020;395(10231):1189-1190

32 Wang D, Hu B, Hu C, et al. Clinical characteristics of 138 hospitalized patients with 2019 novel corona virus infection pneumonia in Wuhan, China. JAMA 2020;323(11):1061-1069

33 Hosseiny M, Kooraki S, Gholamrezanezhad A, Reddy S, Myers L. Radiology perspective of corona virus disease 2019 (COVID-19): lessons from severe acute respiratory syndrome and Middle East respiratory syndrome. AJR Am J Roentgenol 2020;214(5):1078-1082

34 Pozzessere C, Rotzinger DC, Ghaye B, Lamoth F, BeigelmanAubry C. Incidentally discovered COVID-19 pneumonia: the role of diagnostic imaging. Eur Radiol 2020. Doi: 10.1007/ s00330-020-06914-6

35 Hope MD, Raptis CA, Shah A, Hammer MM, Henry TS; six signatories. A role for CT in COVID-19? What data really tell us so far. Lancet 2020;395(10231):1189-1190

36 Andrea L. Caution about radiologic diagnosis of COVID-19 infection driven by artificial intelligence. Lancet 2020;2:e225 